\title{
Functionals linear in curvature and statistics of helical proteins
}

\author{
A. Feoli* \\ Dipartimento di Ingegneria, Università del Sannio, \\ Corso Garibaldi n. 10\%, Palazzo Bosco Lucarelli, \\ 82100 Benevento, Italy \\ and INFN Sezione di Napoli, Gruppo collegato di Salerno \\ 80126 Napoli, Italy \\ V.V. Nesterenko ${ }^{\dagger}$ \\ Bogoliubov Laboratory of Theoretical Physics \\ Joint Institute for Nuclear Research \\ 141980 Dubna, Russia \\ G. Scarpetta ${ }^{\ddagger}$ \\ Dipartimento di Fisica "E.R.Caianiello" - Università di Salerno \\ 84081 Baronissi (SA), Italy \\ and INFN Sezione di Napoli, Gruppo collegato di Salerno \\ 80126 Napoli, Italy
}

\begin{abstract}
The effective free energy of globular protein chain is considered to be a functional defined on smooth curves in three dimensional Euclidean space. From the requirement of geometrical invariance, together with basic facts on conformation of helical proteins and dynamical characteristics of the protein chains, we are able to determine, in a unique way, the exact form of the free energy functional. Namely, the free energy density should be a linear function of the curvature
\end{abstract}

*E-mail: feoli@unisannio.it; fax: +39089965275

${ }^{\dagger}$ E-mail: nestr@thsun1.jinr.ru

${ }^{\ddagger}$ E-mail: scarpetta@sa.infn.it 
of curves on which the free energy functional is defined. We briefly discuss the possibility of using the model proposed in Monte Carlo simulations of exhaustive searching the native stable state of the protein chain. The relation of the model proposed to the rigid relativistic particles and strings is also considered.

PACS numbers: 87.15.-v, 87.15.Cc, 05.10.Ln, 02.40.-k

Keywords: protein folding, semi-flexible polymers, geometry of curves, helices, rigid string, particles with rigidity. 


\section{Introduction}

A fascinating and open question challenging physics, biochemistry and even geometry is the presence of highly regular motifs such as $\alpha$-helices and $\beta$ - sheets in the folded state of biopolymers and proteins. A wide range of approaches have been proposed to rationalize the existence of such secondary structures (see, for example, reviews [1, 2, 3] and references therein).

In principle, in order to find the stable native state of a protein, one should compute, for every possible conformation of the chain, the sum of the free energies of the atomic interactions within the protein as well as with the solvent and then find the conformation with the lowest free energy. However, it is not feasible, because the number of conformations of a protein chain grows exponentially with the chain length.

In the present paper we are going to propose a pure geometrical approach to describe the effective free energy of proteins, proceeding from the most general invariance requirements and basic experimental facts concerning the protein conformation. It should be noted here that we shall consider not the Hamiltonian $H$ (or energy) of an individual protein molecule, but the effective free energy of an assembly of noninteracting protein chains. We define this energy as such a functional $F$ depending on the radius vector $\mathbf{x}(s)$ of a protein chain, the stationary values of which (the extrema) are provided only by the observed configurations of proteins. Obviously, the effective free energy defined in such a way is analogous to the effective action in quantum field theory [4].

Let $E[\mathbf{x}(s)]$ be the energy of an individual protein chain then the effective free energy $F$ we are interested in is apparently determined by the functional integral

$$
e^{-\beta F\left[\mathbf{x}_{c l}\right]}=\int D \mathbf{x}_{q u} e^{-\beta E\left[\mathbf{x}_{c l}+\mathbf{x}_{q u}\right]}
$$

where $\beta$ is inverse temperature and we have split the protein radius vector $\mathbf{x}$ into the classical part $\mathbf{x}_{c l}$ and the quantum part $\mathbf{x}_{q u}$. In order to use this formula the energy functional $E$ for an individual protein chain should be specified. The problem under consideration is close to the statistics of semi-flexible polymers. In the last case a number of models (i.e., particular functionals $E$ ) have been proposed which substantially rely on the differential geometry (Kratky-Porod model [5], Dirac model [6, 7], tube model [8] and so on). Differential geometry provides a direct relation between physics and mathematics and thereby opens a straight route for applying the mathematical analysis to this physical problem [9, 10. If $k(s)$ and $\kappa(s)$ are respectively the curvature and the torsion of a polymer chain then the energy $E$ can be represented as a polynomial in these geometrical invariants, for example, in 
the form 9

$$
E=\int_{0}^{L} d s\left[\frac{A}{2} k^{2}+\frac{C}{4} k^{4}-\alpha k^{2} \kappa+\frac{\beta}{2} k^{2} \kappa^{2}\right],
$$

where $A, C, \alpha$, and $\beta$ are phenomenological parameters.

However evaluation of the functional integral in Eq. (1.1) with the energy $E$ given in Eq. (1.2) or by analogous formula is a nontrivial mathematical problem. The progress on this line is possible only when applying approximate methods 9]. As a rule, the results obtained here do not allow one to predict the exact stationary form of protein or polymer chains, at best one can calculate the statistical momenta of the chains.

In this situation we propose another way of consideration. We shall try straight away to determine the effective free energy $F\left[\mathbf{x}_{c l}\right]$ without specifying the energy functional $E$ of an individual protein chain. For this purpose we shall use experimental knowledge concerning the protein chain configurations and the tools of differential geometry.

Such a statement of the problem on describing the statistical properties of proteins semi-flexible polymers is non-standard one. In some sense it is an inverse with respect to a traditional formulation of the problem. Nevertheless we suppose that such an inverse task is undoubtedly of substantial interest. It turns out that when confining oneself to helical proteins the inverse problem at hand has a nice mathematical solution, being unique. It is the prove of this assertion that is the basic result of this paper.

Taking into account the one-dimensional nature of the protein chains, the relevant macroscopic free energy $F$ should be considered as a functional defined on smooth curves $\mathbf{x}(s)$ (or paths) in the three dimensional Euclidean space

$$
F=\int \mathcal{F}[\mathbf{x}(s)] d s,
$$

where $s$ is the length of a protein molecule. For shortennig the formulas we drop the subscript $c l$ in $\mathbf{x}$. The reparametrization invariance of the functional $F$ demands the free energy density $\mathcal{F}$ to be a scalar function depending on the geometrical invariants of the position vector $\mathbf{x}(s)$, which describes the spatial shape of the protein chain. In three dimensional ambient space a smooth curve has two local invariants: curvature $k(s)$ and torsion $\kappa(s)$. In the general case of $D$ dimensional Euclidean embedding space there are $D-1$ principal curvatures $k_{\alpha}(s), \alpha=1,2 \ldots, D-1$ of a curve, where $k_{1}(s)=k(s)$ and $k_{2}(s)=\kappa(s)$.

The basic theorem (due to O. Bonnet) in the theory of curves reads [11]: given the curvatures $k_{\alpha}(s), \alpha=1,2 \ldots, D-1$, a curve $\mathbf{x}(s)$ is determined in 
$D$-dimensional Euclidean space up to its rotations and displacements as a whole. The curvature $k_{\alpha}(s)$ is expressed in terms of the derivatives, with respect to $s$, of the radius vector of a curve $\mathbf{x}(s)$ till the $(\alpha+1)$-th order included.

The first principal curvature, or simply the curvature, $k_{1}(s)=k(s)$ of a curve characterizes the local bending of the curve at the point $s$. Hence, the dependence of free energy density $\mathcal{F}$ on $k(s)$ specifies the resistance of a protein chain to be bent. The second curvature or torsion $\kappa(s)$ is determined by the relative rotation, around the tangent $d \mathbf{x}(s) / d s$ at the point $s$, of two neighbor infinitely short elements of the protein chain. It is well known [1] that, in the case of protein molecules, such a rotation is quite easy, as it requires little effort. In other words, this rotation results in small energy differences, allowing many overall conformations of a protein chain to arise. Thus the dependence of the free energy density $\mathcal{F}$ on torsion $\kappa(s)$ can be neglected at least as a first approximation. Some comments concerning this restriction will be given in Section 4 . Finally one can consider the free energy density $\mathcal{F}$ to be a function only of the curvature $k(s)$, i.e. $\mathcal{F}=\mathcal{F}(k(s))$. In what follows we shall try to specify this dependence explicitly keeping in mind the description of globular protein conformation.

A peculiarity of conformation of globular proteins is that they can be ordered assemblies either of helices or of sheets as well as a mixture of helices and sheets [1]. In the phenomenological macroscopic approach, which is developed here, the presence of sheets in the spatial structure of globular proteins implies the necessity to introduce, in addition to space curves $\mathbf{x}(s)$, new dynamical variables $\mathbf{y}\left(s, s^{\prime}\right)$ describing surfaces in ambient space. ${ }^{1}$ Obviously such an extension of the problem setting would complicate considerably our consideration. Therefore we confine ourselves to helical proteins and try to answer the question: Is it possible to specify the function $\mathcal{F}(k(s))$ in such a way that the extremals of the functional $F=\int \mathcal{F} d s$ would be only helices? The answer to this question turns out to be positive and unique, namely, the density of the free energy $\mathcal{F}(k(s))$ should be a linear function of the curvature $k(s)$ :

$$
\mathcal{F}(k)=\alpha-|\beta| k(s),
$$

where the constants $\alpha$ and $\beta$ are the parameters specifying the phenomenological model proposed, $\alpha>0, \beta<0$. A rigorous proof of this assertion is the main result of the present paper.

The layout of the paper is as follows. In Section 2 the Euler-Lagrange

\footnotetext{
${ }^{1}$ We do not consider here such exotic curves as Peano's curve which, being specified by one parameter $s$, nevertheless covers, completely tightly, a finite region of a surface (see, for example, Ref. [12]).
} 
variational equations for the functional $F$ are reformulated in terms of the principal curvatures. In Section 3 the explicit integrability of these equations will be shown. In other words, for an arbitrary function $\mathcal{F}(k(s))$, the corresponding Euler-Lagrange equations are integrable by quadratures. The physical meaning of the relevant constants of integrations is also discussed. In Section 4 the requirement that the solutions of the Euler-Lagrange equations describe only helices immediately confines the admissible free energy density $\mathcal{F}(k(s))$ to be a linear function (1.4). In conclusion (Section 5), the obtained results and their physical implications are briefly discussed.

\section{Euler-Lagrange equations in terms of prin- cipal curvatures}

Keeping in mind the description of the protein conformation, one should consider the problem in three dimensional Euclidean space. However, from the mathematical standpoint, it is convenient to formulate the equations we are looking for, first in D-dimensional Euclidean space and only after that to go to the three dimensional ambient space.

We shall follow our papers [13, 14, where the analogous problem has been considered in Minkowski space-time and the results obtained have been applied to the models of relativistic particles described by the Lagrangians dependent on higher derivatives of the particle coordinates. Unfortunately, the transition to the Euclidean space in the final formulas of Ref. [13] is not obvious. $^{2}$ Therefore we derive the equations for the principal curvatures and the relevant integrals of motion in Euclidean space anew. In this section we shall use a natural parametrization of the curve $\mathbf{x}(\mathbf{s})$ in the $D$-dimensional Euclidean space: $x^{i}(s), i=1,2, \ldots, D$. In this parametrization

$$
\frac{d x^{i}}{d s} \frac{d x^{i}}{d s}=\left(\mathbf{x}^{\prime} \mathbf{x}^{\prime}\right)=1
$$

or in another way $d s^{2}=d x^{i} d x^{i}=(d \mathbf{x} d \mathbf{x})$. As usual the sum over the repeated indexes is assumed in the corresponding range and, for shortening, the differentiation with respect to the natural parameter $s$ will be denoted by a prime.

The curve $x^{i}(s), i=1,2, \ldots, D$ has $D-1$ principal (or external) curvatures which determine this curve up to its motion as a whole in embedding

\footnotetext{
${ }^{2}$ Differential geometries of curves in Euclidean space and in Minkowski space-time differ substantially. In main part it is due to three types of curves existing in pseudo-Euclidean space: timelike, spacelike and isotropic (null) curves [15].
} 
space. The first curvature, or simply curvature, is given by

$$
k_{1}^{2}(s)=k^{2}(s)=\frac{d^{2} x^{i}}{d s^{2}} \frac{d^{2} x^{i}}{d s^{2}}=\left(\mathbf{x}^{\prime \prime} \mathbf{x}^{\prime \prime}\right) .
$$

For an arbitrary functional $F$ defined on curves $x^{i}(s)$ in $D$-dimensional space the Euler-Lagrange equations are a set of exactly $D$ equations

$$
\frac{\delta F}{\delta x^{i}}=0, \quad i=1,2, \ldots, D .
$$

However, if the functional $F$ depends only on the curvature

$$
F=\int \mathcal{F}(k(s)) d s
$$

then $D$ equations (2.3) for $D$ variables $x^{i}(s), i=1,2, \ldots, D$ give $D-1$ equations for the principal curvatures $k_{\alpha}(s), \alpha=1,2, \ldots, D-1$.

When deriving the equations for $k_{\alpha}(s)$ we shall use the orthonormal Frenet basis $\left\{\mathbf{e}_{a}\right\}$ :

$$
\left(\mathbf{e}_{a} \mathbf{e}_{b}\right)=\delta_{a b}, \quad a, b=1,2 \ldots ., D
$$

associated with the curve $\mathbf{x}(s)$ and the Frenet equations governing the motion of this basis along the curve $\mathbf{x}(s)$

$$
\frac{d \mathbf{e}_{a}}{d s}=\omega_{a b} \mathbf{e}_{b}, \quad \omega_{a b}+\omega_{b a}=0 .
$$

The unit vector of the Frenet basis $\mathbf{e}_{\alpha}(s)$ is expressed in terms of the derivatives of the position vector $\mathbf{x}(s)$ with respect to the parameter $s$ till the order $\alpha$ included, $\alpha=1,2, \ldots, D$. The first vector $\mathbf{e}_{1}$ is directed along the tangent of the curve at the point $s$

$$
\mathbf{e}_{1}(s)=\frac{d \mathbf{x}}{d s}=\mathbf{x}^{\prime}
$$

In what follows, the explicit expressions, in terms of $\mathbf{x}(s)$, for the rest of the unit vectors $\mathbf{e}_{a}(s)$ are not necessary (relevant formulas can be found, for example, in [16, 17]). Nonzero elements of the matrix $\omega$ in the Frenet equations (2.6) are determined by the principal curvatures

$$
\omega_{\alpha, \alpha+1}=-\omega_{\alpha+1, \alpha}=k_{\alpha+1}(s), \quad \alpha=1, \ldots, D-1 .
$$

In turn, the principal curvature $k_{\alpha}(s)$ is expressed in terms of the derivatives of the position vector $\mathbf{x}(s)$ till the $(\alpha+1)$-th order included. The first 
curvature is defined in Eq. (2.2). Besides, we shall use the explicit expression, through the derivatives of $\mathbf{x}(s)$, only for the second curvature or torsion $k_{2}(s)=\kappa(s)$.

The variation of the space form of the protein molecule

$$
\delta \mathbf{x}(s)=\varepsilon_{a}(s) \mathbf{e}_{a}(s), \quad a=1, \ldots, D
$$

results in the following variation of the free energy functional $F$ defined in Eq. (2.4)

$$
\delta F=\delta F_{1}+\delta F_{2}
$$

where

$$
\begin{gathered}
\delta F_{1}=\int d s \mathcal{F}^{\prime}\left(k_{1}\right) \delta k_{1}(s), \\
\delta F_{2}=\int \mathcal{F}\left(k_{1}\right) \delta d s .
\end{gathered}
$$

In what follows the prime on the free energy density $\mathcal{F}\left(k_{1}\right)$ will denote the differentiation with respect to its argument $k_{1}$.

The variation $\delta d s$ is calculated in a straightforward way

$$
\delta d s=\delta \sqrt{d x^{i} d x^{i}}=\frac{d x^{i} \delta d x^{i}}{d s}=\frac{d x^{i}}{d s} d\left(\delta x^{i}\right)=\left(\mathbf{x}^{\prime} d \delta \mathbf{x}\right) .
$$

The substitution of Eq. (2.13) into $\delta F_{2}$ and the subsequent integration by parts $^{3}$ give

$$
\delta F_{2}=-\int \mathcal{F}^{\prime}\left(k_{1}\right) k_{1}^{\prime}\left(\mathbf{x}^{\prime} \delta \mathbf{x}\right) d s-\int \mathcal{F}\left(k_{1}\right)\left(\mathbf{x}^{\prime \prime} \delta \mathbf{x}\right) d s
$$

By making use of the Frenet equations (2.6) and the expansion (2.9) one can derive

$$
\begin{gathered}
\left(\mathbf{x}^{\prime} \delta \mathbf{x}\right)=\left(\mathbf{e}_{1} \delta \mathbf{x}\right)=\varepsilon_{a}\left(\mathbf{e}_{1} \mathbf{e}_{a}\right)=\varepsilon_{1}, \\
\left(\mathbf{x}^{\prime \prime} \delta \mathbf{x}\right)=\left(\mathbf{e}_{1}^{\prime} \delta \mathbf{x}\right)=\omega_{12}\left(\mathbf{e}_{2} \delta \mathbf{x}\right)=k_{1} \varepsilon_{2} .
\end{gathered}
$$

In view of this, Eq. (2.12) acquires the form

$$
\delta F_{2}=-\int \mathcal{F}^{\prime}\left(k_{1}\right) k_{1}^{\prime} \varepsilon_{1}(s) d s-\int \mathcal{F}\left(k_{1}\right) k_{1}(s) \varepsilon_{2}(s) d s .
$$

\footnotetext{
${ }^{3} \mathrm{We}$ are interested in statistical applications of the ultimate equations, therefore the contributions depending on the position of the protein edges are dropped.
} 
The calculation of the variation $\delta F_{1}$, defined in Eq. (2.11), is more complicated. First we find the variation of the curvature $\delta k_{1}(s)$. Definition $(2.2)$ and the Frenet equations (2.6) give

$$
k_{1}(s) \delta k_{1}(s)=\left(\mathbf{x}^{\prime \prime} \delta \mathbf{x}^{\prime \prime}\right)=\left(\mathbf{e}_{1}^{\prime} \delta \mathbf{x}^{\prime \prime}\right)=k_{1}\left(\mathbf{e}_{2} \delta \mathbf{x}^{\prime \prime}\right) .
$$

Hence

$$
\delta k_{1}=\left(\mathbf{e}_{2} \delta \mathbf{x}^{\prime \prime}\right) .
$$

Applying Eq. (2.13) step by step one derives

$$
\begin{aligned}
& \delta \mathbf{x}^{\prime}=\delta \frac{d \mathbf{x}}{d s}=\frac{d}{d s} \delta \mathbf{x}-\frac{d \mathbf{x}\left(\mathbf{x}^{\prime} d \delta \mathbf{x}\right)}{d s^{2}}=\frac{d}{d s} \delta \mathbf{x}-\mathbf{x}^{\prime}\left(\mathbf{x}^{\prime} \frac{d}{d s} \delta \mathbf{x}\right)=\frac{d}{d s} \delta \mathbf{x}-\mathbf{e}_{1}\left(\mathbf{e}_{1} \frac{d}{d s} \delta \mathbf{x}\right), \\
& \delta \mathbf{x}^{\prime \prime}=\delta \frac{d \mathbf{x}^{\prime}}{d s}=\frac{d}{d s} \delta \mathbf{x}^{\prime}-\frac{d \mathbf{x}^{\prime}\left(\mathbf{x}^{\prime} d \delta \mathbf{x}\right)}{d s^{2}}=\frac{d^{2}}{d s^{2}} \delta \mathbf{x}-2 \mathbf{e}_{1}^{\prime}\left(\mathbf{e}_{1} \frac{d}{d s} \delta \mathbf{x}\right)-\mathbf{e}_{1} \frac{d}{d s}\left(\mathbf{e}_{1} \frac{d}{d s} \delta \mathbf{x}\right) .
\end{aligned}
$$

In view of (2.17), the last term in Eq. (2.19) does not contribute to $\delta k_{1}$. Substituting Eq. (2.19) into (2.17) and using the Frenet equations (2.6) together with expansion (2.9) we obtain

$$
\delta k_{1}(s)=\varepsilon_{1} k_{1}^{\prime}+\varepsilon_{2}+\varepsilon_{2}\left(k_{1}^{2}-k_{2}^{2}\right)-2 \varepsilon_{3}^{\prime} k_{2}-\varepsilon_{3} k_{2}^{\prime}-\varepsilon_{4} k_{2} k_{3} .
$$

As one could expect formula (2.20) for $\delta k_{1}$ differs from its analogue in Minkowski space (see Eq. (2.18) in Ref. [13] only by signs of some terms. However, without the direct calculation of $\delta k_{1}$ in Euclidean space the rule of sign changes in this expression is not obvious.

Thus, the variation of the first curvature, $\delta k_{1}(s)$, depends on the variations of the worldline coordinates only along the directions $\mathbf{e}_{1}, \mathbf{e}_{2}, \mathbf{e}_{3}$, and $\mathbf{e}_{4}$ (on the four arbitrary functions $\left.\varepsilon_{a}(s), a=1,2,3,4\right)$ and on the first three curvatures $k_{1}, k_{2}$, and $k_{3}$.

Substituting Eq. (2.20) into (2.11), integrating the latter equation by parts, and taking into account (2.16) we obtain

$$
\begin{aligned}
\delta F & =\int d s\left\{\left[\left(k_{1}^{2}-k_{2}^{2}\right) \mathcal{F}^{\prime}\left(k_{1}\right)+\frac{d^{2}}{d s^{2}}\left(\mathcal{F}^{\prime}\left(k_{1}\right)\right)-k_{1} \mathcal{F}\left(k_{1}\right)\right] \varepsilon_{2}(s)\right. \\
& \left.+\left[2 \frac{d}{d s}\left(\mathcal{F}^{\prime}\left(k_{1}\right) k_{2}\right)-k_{2}^{\prime} \mathcal{F}^{\prime}\left(k_{1}\right)\right] \varepsilon_{3}(s)-\mathcal{F}^{\prime}\left(k_{1}\right) k_{2} k_{3} \varepsilon_{4}(s)\right\}=0 .
\end{aligned}
$$

The terms in $\delta F_{1}$ and $\delta F_{2}$, depending on the variation $\varepsilon_{1}(s)$ along the tangent to the curve, are mutually cancelled, and the variation $\delta F$ depends, as one could expect, only on the normal variation of the curve $\mathbf{x}(s)$, or more 
precisely, on the variation of $\mathbf{x}(s)$ along the three normals $\mathbf{e}_{2}, \mathbf{e}_{3}$, and $\mathbf{e}_{4}$. Apparently the last property of the variation $\delta F$ is due to a special type of functionals (2.4) under consideration, which depend only on the curvature of a curve. From Eq. (2.21) three equations for principal curvatures follow

$$
\begin{aligned}
\frac{d^{2}}{d s^{2}}\left(\mathcal{F}^{\prime}\left(k_{1}\right)\right) & =-\left(k_{1}^{2}-k_{2}^{2}\right) \mathcal{F}^{\prime}\left(k_{1}\right)+k_{1} \mathcal{F}\left(k_{1}\right), \\
2 \frac{d}{d s}\left(\mathcal{F}^{\prime}\left(k_{1}\right) k_{2}\right) & =k_{2}^{\prime} \mathcal{F}^{\prime}\left(k_{1}\right), \\
\mathcal{F}^{\prime}\left(k_{1}\right) k_{2} k_{3} & =0 .
\end{aligned}
$$

At first glance, Eqs. (2.22) - (2.24) are obviously insufficient in order to determine all $D-1$ principal curvatures of the curve $\mathbf{x}(s)$ embedded in $D$ dimensional Euclidean space with arbitrary $D$. However, it is not the case.

We start the analysis of the obtained equations from Eq. (2.24). The most general condition on the principal curvatures, following from this equation, is the requirement of vanishing the third curvature

$$
k_{3}(s)=0 \text {. }
$$

The point is that the principal curvatures, due to their construction, obey the conditions [17, 18: if a curvature $k_{j}(s)$ does not vanish at the point $s$, then at this point the curvatures $k_{\alpha}(s)$ with $\alpha=1,2, \ldots, j-1$ are also different from zero. But the vanishing of $k_{j}(s)$ at a point $s, k_{j}(s)=0$, implies that $k_{\alpha}(s)=0$ for $\alpha=j+1, j+2, \ldots, D-1$. In view of this, Eq. (2.25) entails the following conditions

$$
k_{4}(s)=k_{5}(s)=\ldots=k_{D-1}(s)=0 .
$$

Thus, in the problem under consideration there are two non trivial equations (2.22) and (2.23) for the curvatures $k_{1}(s)$ and $k_{2}(s)$. Equation (2.23) can be integrated with arbitrary free energy density $\mathcal{F}\left(k_{1}\right)$. Actually, for $k_{2} \neq 0$ one can rewrite this equation in the form

$$
\frac{k_{2}^{\prime}}{k_{2}}+2 \frac{\mathcal{F}^{\prime \prime}\left(k_{1}\right) k_{1}^{\prime}}{\mathcal{F}^{\prime}\left(k_{1}\right)}=0
$$

or

$$
2 d\left[\ln \mathcal{F}^{\prime}\left(k_{1}\right)\right]+d\left(\ln k_{2}\right)=0 .
$$

Hence

$$
\left(\mathcal{F}^{\prime}\left(k_{1}\right)\right)^{2} k_{2}=C,
$$

where $C$ is an integration constant. 
Relation (2.27) enables one to eliminate the torsion $k_{2}(s)$ from Eq. (2.22). As a result we are left with one nonlinear differential equation of the second order for the curvature $k_{1}(s)$

$$
\frac{d^{2}}{d s^{2}} \mathcal{F}^{\prime}\left(k_{1}\right)+\left(k_{1}^{2}-\frac{C^{2}}{\left(\mathcal{F}^{\prime}\left(k_{1}\right)\right)^{4}}\right) \mathcal{F}^{\prime}\left(k_{1}\right)-k_{1} \mathcal{F}\left(k_{1}\right)=0
$$

Having resolved this equation for $k_{1}(s)$, one can determine the rest of curvatures by making use of Eqs. (2.27), (2.25), and (2.26). Integration of the Frenet equations (2.6) with principal curvatures found enables one to recover the curve $\mathbf{x}(s)$ itself.

Notwithstanding its nonlinear character, Eq. (2.28) can be integrated in quadratures for arbitrary function $\mathcal{F}\left(k_{1}\right)$. To show this, the first integral for this equation will be constructed proceeding from the symmetry properties of the variational problem under study.

\section{Exact integrability of the Euler-Lagrange equations for principal curvatures}

The functional (2.4) possesses a quite large set of symmetries, the analysis of which will allow us to construct the first integral for nonlinear equation (2.28). In this section we shall use an arbitrary parametrization of the curve $\mathbf{x}(\tau)$. Now the functional (2.4) assumes the form

$$
F=\int \mathcal{F}\left(k_{1}\right) \sqrt{\dot{\mathbf{x}}^{2}} d \tau
$$

where a dot over $\mathbf{x}$ denotes the differentiation with respect to the parameter $\tau$. In arbitrary parametrization the curvature $k_{1}$ is given by

$$
k_{1}^{2}=\frac{\dot{\mathbf{x}}^{2} \ddot{\mathbf{x}}^{2}-(\dot{\mathbf{x}} \ddot{\mathbf{x}})^{2}}{\left(\dot{\mathbf{x}}^{2}\right)^{3}} \text {. }
$$

Functional (3.1) is invariant under the following transformations:

i) translations of the curve coordinates by a constant vector

$$
\mathbf{x} \rightarrow \mathbf{x}+\mathbf{a}, \quad \mathbf{a}=\text { const }
$$

ii) $S O(D)$-rotations of the ambient space coordinates

$$
x^{i} \rightarrow x^{i}+\omega^{i j} x^{j}, \quad \omega^{i j}=-\omega^{j i}, \quad i, j=1,2, \ldots, D
$$


iii) reparametrization

$$
\tau \rightarrow f(\tau)
$$

with an arbitrary function $f(\tau)$ subjected to the condition $\dot{f}(\tau) \neq 0$.

According to the first Noether theorem, the invariance of the functional (3.1) under the translations (3.3) entails the conservation, under the motion along the curve $\mathbf{x}(s)$, of the 'momentum' vector

$$
P^{i}=\frac{d}{d \tau}\left(\frac{\partial \sqrt{\dot{\mathbf{x}}^{2}} \mathcal{F}\left(k_{1}\right)}{\partial \ddot{x}^{i}}\right)-\frac{\partial \sqrt{\dot{\mathbf{x}}^{2}} \mathcal{F}\left(k_{1}\right)}{\partial \dot{x}^{i}} \equiv P_{0}^{i}, \quad i=1,2, \ldots, D,
$$

where $P_{0}^{i}$ are constants. The Euler-Lagrange equations (2.3), rewritten in terms of $P^{i}$, exhibit this manifestly

$$
\frac{d}{d \tau} P^{i}=0, \quad i=1,2, \ldots, D
$$

It is clear that the relations (3.6), due to their vector character, can not be used directly as integrals for Eqs. (2.22)-(2.24) determining the principal curvature $k_{\alpha}$. Simply, the vector $P^{i}$ can not be expressed only in terms of the invariants $k_{\alpha}(s)$. However, one can hope that the invariant

$$
\mathbf{P}^{2}=\mathbf{P}_{0}^{2} \equiv M^{2}
$$

where $M^{2}$ is a new non negative constant, can be expressed through the principal curvatures $k_{\alpha}(s)$ only. It turns out that this is really the case.

To show this, we pass in Eqs. (3.6) from the differentiation with respect to arbitrary parameter $\tau$ to the differentiation with respect to the natural parameter $s$ (the length of a curve). For this purpose the formulas

$$
\begin{gathered}
\frac{d}{d \tau}=\sqrt{\dot{\mathbf{x}}^{2}} \frac{d}{d s}, \quad \frac{d^{2} \mathbf{x}}{d s^{2}}=\frac{\dot{\mathbf{x}}^{2} \ddot{\mathbf{x}}-(\dot{\mathbf{x}} \ddot{\mathbf{x}}) \dot{\mathbf{x}}}{\left(\dot{\mathbf{x}}^{2}\right)^{2}}, \quad k_{1} \frac{\partial k_{1}}{\partial \ddot{\mathbf{x}}}=\frac{1}{\dot{\mathbf{x}}^{2}} \frac{d^{2} \mathbf{x}}{d s^{2}}, \\
k_{1} \frac{\partial k_{1}}{\partial \dot{\mathbf{x}}}=\frac{1}{\left(\dot{\mathbf{x}}^{2}\right)^{4}}\left\{\left[3(\dot{\mathbf{x}} \ddot{\mathbf{x}})^{2}-2 \dot{\mathbf{x}}^{2} \ddot{\mathbf{x}}^{2}\right] \dot{\mathbf{x}}-\dot{\mathbf{x}}^{2}(\dot{\mathbf{x}} \ddot{\mathbf{x}}) \ddot{\mathbf{x}}\right\}
\end{gathered}
$$

are useful. As a result, Eq. (3.6) for the vector $\mathbf{P}$, constant along the curve $\mathbf{x}(s)$, acquires the form

$$
\mathbf{P}=\left[2 \mathcal{F}^{\prime}\left(k_{1}\right) k_{1}-\mathcal{F}\left(k_{1}\right)\right] \frac{d \mathbf{x}}{d s}+\frac{d}{d s}\left(\frac{\mathcal{F}^{\prime}\left(k_{1}\right)}{k_{1}}\right) \frac{d^{2} \mathbf{x}}{d s^{2}}+\frac{\mathcal{F}^{\prime}\left(k_{1}\right)}{k_{1}} \frac{d^{3} \mathbf{x}}{d s^{3}} .
$$


The definitions of the parameter $s$ (2.1) and the curvature $k_{1}(2.2)$ entail the relations

$$
\left(\frac{d \mathbf{x}}{d s} \frac{d^{2} \mathbf{x}}{d s^{2}}\right)=0, \quad\left(\frac{d \mathbf{x}}{d s} \frac{d^{3} \mathbf{x}}{d s^{3}}\right)=-k_{1}^{2}, \quad\left(\frac{d^{2} \mathbf{x}}{d s^{2}} \frac{d^{3} \mathbf{x}}{d s^{3}}\right)=k_{1} \frac{d k_{1}}{d s} .
$$

The vector $\mathbf{x}^{\prime \prime \prime}$ squared can be deduced from the definition of the second curvature $k_{2}$ or torsion [11, 16]

$$
k_{1}^{4} k_{2}^{2}=\operatorname{det}_{G}\left(\frac{d \mathbf{x}}{d s}, \frac{d^{2} \mathbf{x}}{d s^{2}}, \frac{d^{3} \mathbf{x}}{d s^{3}}\right),
$$

where $\operatorname{det}_{G}(\mathbf{a}, \mathbf{b}, \mathbf{c})$ is the Gramm determinant for vectors $\mathbf{a}, \mathbf{b}$, and $\mathbf{c}$ [19]. Thanks to Eq. (2.27), we obtain from (3.12)

$$
\left(\frac{d^{3} \mathbf{x}}{d s^{3}}\right)^{2}=k_{1}^{4}+\left(\frac{d k_{1}}{d s}\right)^{2}+k_{1}^{2} k_{2}^{2}=k_{1}^{4}+\left(\frac{d k_{1}}{d s}\right)^{2}+k_{1}^{2} \frac{C^{2}}{\left(\mathcal{F}^{\prime}\left(k_{1}\right)\right)^{4}} .
$$

Squaring Eq. (3.10) and using Eqs. (3.11) and (3.13), we have

$$
M^{2}=\left(\mathcal{F}^{\prime}\left(k_{1}\right) k_{1}-\mathcal{F}\left(k_{1}\right)\right)^{2}+\frac{C^{2}}{\left(\mathcal{F}^{\prime}\left(k_{1}\right)\right)^{2}}+\left(k_{1}^{\prime}\right)^{2}\left(\mathcal{F}^{\prime \prime}\left(k_{1}\right)\right)^{2} .
$$

In contrast to pseudo-Euclidean space [13, the right-hand side of Eq. (3.14) is strictly nonnegative.

By direct differentiation of Eq. (3.14) with respect to $s$ one can be convinced that, for

$$
\mathcal{F}^{\prime \prime}\left(k_{1}\right) \neq 0
$$

the relation (3.14) is an integral of the nonlinear differential equation (2.28), which determines the curvature of a stationary curve. From (3.14) we deduce

$$
\frac{d k_{1}}{d s}= \pm \sqrt{f\left(k_{1}\right)}
$$

where

$$
f\left(k_{1}\right)=\frac{1}{\left(\mathcal{F}^{\prime \prime}\left(k_{1}\right)\right)^{2}}\left[M^{2}-\frac{C^{2}}{\left(\mathcal{F}^{\prime}\left(k_{1}\right)\right)^{2}}-\left(k_{1} \mathcal{F}^{\prime}\left(k_{1}\right)-\mathcal{F}\left(k_{1}\right)\right)^{2}\right] .
$$

Integration of (3.16) gives

$$
\int_{k_{10}}^{k_{1}(s)} \frac{d k}{\sqrt{f(k)}}= \pm\left(s-s_{0}\right)
$$

with $k_{10}=k_{1}\left(s_{0}\right)$. 
Thus, if the free energy density $\mathcal{F}\left(k_{1}\right)$ obeys the condition (3.15), then the curvature $k_{1}(s)$ and the torsion $k_{2}(s)$ of the stationary curve $\mathbf{x}(s)$ are the functions of the parameter $s$ defined by Eqs. (3.18) and (2.27). The case when the condition (3.15) is not satisfied, i.e., when the free energy density $\mathcal{F}\left(k_{1}\right)$ is a linear function of the curvature $k_{1}(s)$, will be considered in the next section.

Closing this section we briefly consider the consequences of the invariance of the free energy under the transformations (3.4) and (3.5). According to the first Noether theorem, the rotation invariance of the functional (2.4) entails the conservation of the angular momentum tensor

$$
M^{i j}=\sum_{\sigma=1}^{2}\left(q_{\sigma}^{i} p_{\sigma}^{j}-q_{\sigma}^{j} p_{\sigma}^{i}\right), \quad i<j,
$$

where the canonical variables $\mathbf{q}_{\sigma}$ and $\mathbf{p}_{\sigma}, \sigma=1,2$ are defined as follows [20]

$$
\begin{gathered}
\mathbf{q}_{1}=\mathbf{x}, \quad \mathbf{q}_{2}=\dot{\mathbf{x}} \\
\mathbf{p}_{1}=\mathbf{P}=-\frac{\partial\left(\sqrt{\dot{\mathbf{x}}^{2}} \mathcal{F}\right)}{\partial \dot{\mathbf{x}}}-\frac{d \mathbf{p}_{2}}{d \tau}, \quad \mathbf{p}_{2}=-\frac{\partial\left(\sqrt{\dot{\mathbf{x}}^{2}} \mathcal{F}\right)}{\partial \ddot{\mathbf{x}}} .
\end{gathered}
$$

As in the case of the translation invariance, the conserved angular momentum tensor (3.19) does not provide by itself the integrals for the equations determining the principal curvatures, while the invariant $S^{2}$ constructed in terms of this tensor does

$$
S^{2}=\frac{W}{M^{2}},
$$

where

$$
W=\frac{1}{2} M^{i j} M^{i j} \mathbf{P}^{2}-\left(M^{i j} P^{j}\right)^{2}, \quad M^{2}=\mathbf{P}^{2} .
$$

In Minkowski space-time the analogous invariant is the spin of a dynamical system 21]. Direct calculation gives [13]

$$
M^{2} S^{2}=k_{2}^{2}\left(\mathcal{F}^{\prime}\right)^{4}
$$

Thus the invariant S specifies the integration constant C in Eq. (2.27), namely

$$
C^{2}=M^{2} S^{2}
$$

According to the second Noether theorem [22], the invariance of the functional (3.1) under the reparametrization (3.5) results in the identity which is obeyed by the left-hand sides of the Euler-Lagrange equations (2.3). As 
in the theory of relativistic strings [23], one can show that the projection of Eqs. (2.3) on the tangent vector $\dot{\mathbf{x}}(\tau)$ is identically equal to zero

$$
\frac{\delta F}{\delta x^{i}} \dot{x}^{i}=0
$$

Thus between equations (2.3) there are only $D-1$ independent ones. It is this fact that explains why we succeeded in obtaining the closed set of $D-1$ equation for the principal curvatures of a stationary curve.

\section{Determining the functional form of the free energy density}

We are going to fix the function $\mathcal{F}\left(k_{1}\right)$, requiring that all the solutions to the Euler - Lagrange equations are helices. From the differential geometry of curves [11, 17] it is known that the helices in three dimensional space have a constant curvature $\left(k_{1}\right)$ and a constant torsion $\left(k_{2}\right)$ which determine the radius $R$ and the step $d$ of a helix ${ }^{4}$

$$
R=\frac{k_{1}}{k_{1}^{2}+k_{2}^{2}}, \quad d=\frac{2 \pi\left|k_{2}\right|}{k_{1}^{2}+k_{2}^{2}}
$$

As shown in the previous section, under the condition (3.15) the curvature $k_{1}$ and the torsion $k_{2}$ of the stationary curve are not constant but they are the functions of the parameter $s$ which are defined in Eqs. (3.18) and (2.27). Hence, for the free energy density $\mathcal{F}\left(k_{1}\right)$ we are looking for, we have to replace Eq. (3.15) with the requirement

$$
\mathcal{F}^{\prime \prime}\left(k_{1}\right)=0
$$

So $\mathcal{F}\left(k_{1}\right)$ should be a linear function of the curvature $k_{1}(s)$

$$
\mathcal{F}\left(k_{1}\right)=\alpha+\beta k_{1}(s),
$$

where $\alpha$ and $\beta$ are constants. Substituting Eq. (4.3) into (2.27) and (2.28) we obtain

$$
k_{1}=-\frac{C^{2}}{\alpha \beta^{3}}, \quad k_{2}=\frac{C}{\beta^{2}} .
$$

\footnotetext{
${ }^{4}$ It is interesting to note that for an arbitrary dimension $D$ of the ambient space, the curves with constant principal curvatures have a drastically different behavior in the large depending on whether the dimension $D$ is even or odd. If $D$ is even, then the curves under consideration are situated in a restricted part of the space. For odd $D$, such curves go to infinite in one direction.
} 
Since $k_{1}(s)=\left|\mathbf{x}^{\prime \prime}(s)\right|>0$, the constants $\alpha$ and $\beta$ should have opposite signs. It is natural to put $\alpha>0$ and $\beta<0$.

For the free energy density $\mathcal{F}\left(k_{1}\right)$, linear in curvature, the integral (3.14) gives just the relation between the integration constants $M^{2}$ and $S^{2}$ (or between $M^{2}$ and $C^{2}$ )

$$
M^{2}=\frac{\alpha^{2}}{1-\beta^{-2} S^{2}}
$$

When $\alpha=0$, Eq. (2.28) implies that the curvature $k_{1}(s)$ is an arbitrary function of $s$ and the integration constant $C$ vanishes. In this case Eq. (2.27) yields $k_{2}=0$. Hence, for $\alpha=0$, the solutions to the Euler-Lagrange equations are arbitrary plane curves, which is evidently unacceptable for our purpose.

Finally, requiring that the stationary curves for the functional (2.4) are only helices, we uniquely determine the free energy density, namely it should be a linear function of the curvature

$$
\mathcal{F}\left(k_{1}\right)=\alpha-|\beta| k_{1}(s)
$$

with nonzero constants $\alpha$ and $\beta$, providing $\alpha>0$ and $\beta<0$.

In order to get some insight into the physical meaning of the invariants $M^{2}$ and $C^{2}$ we calculate them for protein chains of different forms. For straight line chain we have

$$
M^{2}=\alpha^{2}, \quad C=0 .
$$

For stationary chains (helices) these invariants are

$$
M^{2}=\alpha^{2}\left[1+\left(\frac{2 \pi R}{d}\right)^{2}\right], \quad C=\alpha \beta \frac{2 \pi R}{d} .
$$

Here it is worth noting the following. As known, in the protein physics the chirality [24] property of these molecules are of importance. At the first glance we have ignored this point because from the very beginning we have eliminated the dependence of the free energy density $f$ on the curve torsion. But the fact is the integration constant $C$ in our equations is responsible for the chirality property of the helical curves under consideration. Really, this constant is an analog of the Pauli-Lubanski pseudoscalar [25, 26] (in the three dimensional space-time the Pauli-Lubanski vector reduces to the pseudoscalar). The positive and negative values of this constant distinguish the left-hand and right-hand chirality of the helical curves.

Recently, the functionals defined on the world trajectories in Minkovski spacetime and depending on the Cartan curvatures of those lines have been 
considered to be a generalization of the relativistic model of a point particle with possible application to string theory [27], boson-fermion transmutations [28, anyon models [29], random walks [30] and so on. It is worth noting that the Lagrange functions linear in a curvature of the world line prove to be distinguished in this field too. The richest set of appealing properties is provided by the Plyushchay model [31, 32, where $\alpha=0$. In this case the action is scale invariant. A complete set of constraints in the phase space of this model possesses many symmetries, specifically, local $W_{3}$ symmetry. This model describes the massless particles, the helicity of which acquires, upon quantization, integer and half-odd-integer values. It is interesting that classical solutions in this model are the spacelike helical curves 31.

\section{Conclusion}

Proceeding from rather general principles and making use of the basic facts concerning the conformation of globular proteins we have obtained, in a unique way, a geometrical model for phenomenological description of the free energy of helical proteins. It is worth noting that our functional (4.6) should be considered as an effective free energy of the helical protein which already takes into account the $\mathrm{n}$ atomic interactions within the protein and with the solvent. Hence, there is no need to quantize it, as one proceeds in the random walk studies 30].

Certainly our simple model does not pretend to describe all the aspects of the protein folding. However, one can hope that it could be employed, for example, in Monte Carlo simulation to search for a stable native state of the protein. In this case the model can be used for the description of the free energy of individual parts (blocks) of a protein chain that have the helical form. Without any doubt, it should result in simplification and acceleration of the exhausting searching of the native stable state of the protein chain by a computer [1].

In the general problem of the protein folding, a self containing task is to reveal the mechanism of the protein chain transition into the stable native state. A typical time of this process is such that it is completely insufficient to show the mutual influence of all the parts of the protein chain during the folding to the stable state. Presumably the functionals proposed in this paper can be useful here too. For this purpose the coordinates of the protein chain $\mathbf{x}$ should be considered as time dependent, i.e., $\mathbf{x}=\mathbf{x}(t, s)$. During its motion, the protein chain sweeps out a two dimensional surface in ambient space described parametrically by its coordinates $\mathbf{x}(t, s)$. In this case the analogue of the line curvature $k(s)$ is the local geometrical invariant of a surface, its 
external or mean curvature $H(t, s)$ [1]. A straightforward generalization of the functional (2.4) to the dynamical problem at hand is the action $S$ linear in external curvature of the surface

$$
S=a \iint d \sigma+b \iint H d \sigma
$$

where $d \sigma$ is a differential element of the surface swept out by a protein chain in the course of its motion, $a$ and $b$ are constants.

In the elementary particle theory the models like (5.1) are known as the rigid relativistic string [23, 33. One of the motivations to consider such strings was the attempt to develop string description for quantum chromodynamics. A peculiarity of the classical dynamics of such string models is its instability 34]. The reason of this is the dependence of the action (5.1) not only on the velocity $d \mathbf{x}(t, s) / d t$ but also on the acceleration $d^{2} \mathbf{x}(t, s) / d t^{2}$ of the protein chain. As a result, the energy of such systems proves to be unbounded from below [35. It is very likely that this instability could be crucial in describing, in the framework of the string model (5.1), the transition of the protein chain to the stable native state.

\section{Acknowledgements}

This study has been conducted during the stay of one of the authors (V.V.N.) at Salerno University. He would like to thank G. Scarpetta, G. Lambiase, and A. Feoli for the hospitality extended to him. One of the authors (A.F.) wishes to thank A. Rampone for useful discussions and references about the protein folding problem. The financial support of INFN is acknowledged. V.V.N. was partially supported by the Russian Foundation for Basic Research (Grant No. 03-01-00025) and by the International Science and Technology Center (Project No. 840).

\section{References}

[1] H.S. Chan and K.A. Dill, Physics Today, 46, No. 2 (1993) 24.

[2] K.A. Dill, Protein Science, 8 (1999) 1166.

[3] F.R. Banavar, A. Maritan, C. Micheletti, and F. Seno, Geometrical aspects of protein folding, cond-mat/0105209.

[4] P. Ramon, Field Theory. A Modern Primer, The Benjamin/Cummings Publishing Company Inc., London, 1981. 
[5] O. Kratky, G. Porod, Recl. Trav. Chim. 68 (1949) 237.

[6] A. Kholodenko, Phys. Lett. A 141 (1989) 351; Ann. Phys. (N. Y.) 202 (1990) 186.

[7] A. Kholodenko, M. Ballauff, M. Aguero Granados, Physica A, 260 (1998) 267.

[8] J.R. Banavar, A. Maritan, Rev. Mod. Phys. 75 (2003) 23.

[9] R.D. Kamien, Rev. Mod. Phys. 74 (2202) 953.

[10] S. Hyde, S. Anderson, K. Larsson, The Language of Shape, Elsevier, Amsterdam, 1997.

[11] L.P. Eisenhart, Riemannian Geometry, Princeton University Press, Princeton, 1964;

M.P. Do Carmo, Differential Geometry of Curves and Surfaces, PrenticeHall, London, 1976;

M. Spivak, A Comprehensive Introduction to Differential Geometry, Publish or Perish Inc., Houston, 1979.

[12] R. Salem and A. Zygmund, Duke Math. J. 12 (1945) 559.

[13] V.V. Nesterenko, A. Feoli and G. Scarpetta, J. Math. Phys. 36 (1995) 5552 .

[14] V.V. Nesterenko, A. Feoli and G. Scarpetta, Class. Quantum Grav. 13 (1996) 1201.

[15] B. O'Neill, Semi - Riemannian Geometry, Academic, New York, London, 1983;

K. L. Duggal and A. Bejancu, Lightlike Submanifolds of Semi - Riemannian Manifolds and Applications, vol. 364 of Mathematics and its Applications, Kluwer, Dordrecht, The Netherlands, 1996.

[16] M.M. Postnikov, Lectures on Geometry. Semester III: Smooth Manifolds, Nauka, Moscow, 1987.

[17] Yu.A. Aminov, Differential Geometry and Topology of Curves, Nauka, Moscow, 1987.

[18] P.A. Griffiths, Exterior differential systems and the calculus of variations, Birkhäuser, Boston, 1983). 
[19] G.A. Korn and T.M. Korn, Mathematical Handbook, McGraw-Hill, New York, 1968.

[20] V.V. Nesterenko, J. Phys. A 22 (1989) 1673.

[21] S.S. Schweber, An Introduction to Relativistic Quantum Field Theory, Row Peterson, New York, 1961.

[22] B.M. Barbashov and V.V. Nesterenko, Fortschritte der Physik, 31 (1983) 535.

[23] B.M. Barbashov and V.V. Nesterenko, Introduction to the Relativistic String Theory, World Scientific, Singapore, 1990.

[24] A.B. Harris, R.D. Kamien, and T.C. Lubensky, Rev. Mod. Phys. 71 (1999) 1745.

[25] V. V. Nesterenko, J. Math. Phys. 32, 3315 (1991).

[26] V. V. Nesterenko, J. Math. Phys. 34, 5589 (1993).

[27] A.M. Polyakov: Nucl. Phys. B 286 (1986) 406;

H. Kleinert, Phys. Lett. B 174 (1986) 335;

V.V. Nesterenko, J. Math. Phys. 32 (1992) 3315;

M.S. Plyushchay, Phys. Lett. B 253 (1991) 50;

H. Arodź, A. Sitarz, and P. Wegrzyn, Acta Phys. Polonica B 20 (1989) 921;

M. Pavsic, Phys. Lett. B 205 (1988) 231;

J. Grundberg, J. Isberg, U. Lindström and H. Nordström, Phys. Lett. B 231 (1989) 61;

J. Isberg, U. Lindström and H. Nordström, Mod. Phys. Lett. A 5 (1990) 2491.

[28] A.M. Polyakov, Mod. Phys. Lett. 3 A (1988)325;

V.V. Nesterenko, Class. Quantum Grav. 9 (1992) 1101;

M.S. Plyushchay, Nucl. Phys. B 362 (1991) 54;

S. Iso, C. Itoi, and H. Mukaida, Nucl. Phys. B 346 (1990) 293.

[29] V.V. Nesterenko, J. Math. Phys. 34 (1993) 5589.

[30] R.D. Pisarski, Phys. Rev. D 34 (1986) 670;

J. Ambjörn, B. Durhuus, and T. Jonsson, J. Phys. A 21 (1988) 981;

A.L. Kholodenko, Ann. Phys. 202 (1990) 186. 
[31] M.S. Plyushchay, Mod. Phys. Lett. A 4 (1988) 837;

M.S. Plyushchay, Int. J. Mod. Phys. A 4 (1989) 3851;

M.S. Plyushchay, Phys. Lett. B 243 (1990) 383.

[32] C. Batlle, J. Gomis, J.M. Pons, and N. Roman-Roy, J. Phys. A: Math. Gen. 21 (1989) 2693.

[33] A.L. Kholodenko and V.V. Nesterenko, J. Geometry and Physics 16 (1995) 15.

[34] T.L. Curtright, G.I. Ghandour, C.B. Torn, and C.K. Zachos, Phys. Rev. Lett. 57 (1986) 799;

T.L. Curtright, G.I. Ghandour, and C.K. Zachos, Phys. Rev. D 57 (1986);

E. Braaten and C.K. Zachos, Phys. Rev. D 35 (1987) 1512;

F. David and E. Guitter, Europhys. Lett. 3 (1987) 1169.

[35] A.M. Chervyakov and V.V. Nesterenko, Phys. Rev. D 48 (1993) 5811. 